\title{
Inverse Determination of Coupling of Modes Parameters of Surface Acoustic Wave Resonators
}

\author{
T.-T. Wu*, S.-M. Wang, Y.-Y. Chen, T.-Y. Wu ${ }^{1}$, P.-Z. Chang, L.-S. HuAng, C.-L. WAng ${ }^{1}$, C.-W. Wu and C.-K. LeE
}

Institute of Applied Mechanics, National Taiwan University, Taipei, Taiwan 106, R.O.C.

${ }^{1}$ TXC Corporation, Tao-Yuan, Taiwan, R.O.C.

(Received May 1, 2002; accepted for publication August 5, 2002)

Extraction of coupling of modes (COM) parameters from the manufacturing process of a surface acoustic wave device (SAW) is important in practice. In this study, we employed an inverse algorithm to recover the COM parameters from the measured admittance of a one-port SAW resonator. For completeness, the COM equations, the P-matrix representation and the related COM parameters have been summarized. The admittance of a one-port resonator was derived based on the P-matrix representation. One-port and two-port SAW resonator filters were fabricated and the related responses were measured. The simplex method was then used to determine the COM parameters inversely. The recovered COM parameters of the one-port SAW resonator have been demonstrated to be applicable to simulate not only the frequency response of the one-port SAW resonator but also that of the two-port one. [DOI: 10.1143/JJAP.41.6610]

KEYWORDS: surface acoustic wave, coupling of modes, IDT, inverse, resonator filter

\section{Introduction}

The development of mobile phone systems has aroused much interest in the investigations of surface acoustic wave (SAW) devices. In the SAW device design, the coupling of modes (COM) model has been used successfully in modeling surface acoustic wave (SAW) devices for many years. ${ }^{1-3)}$ In the COM analysis, parameters of the COM differential equations are obtained either by measurements or by borrowing from some related theoretical modeling. ${ }^{4-6)}$ COM parameters computed from the theoretical model have provided excellent guidelines for the design of SAW filters, nevertheless, experimental values of the COM parameters are still very important in practice to realize high precision. Although there is some literature on the analysis of various types of SAW filters, literature on the measurements of COM parameters are limited. In the past, Hartmann and Hartmann ${ }^{7)}$ used a five-transducer test structure for measuring COM parameters of Rayleigh-type SAW. Later, Hartmann and Plessky ${ }^{8)}$ applied the same test structure to measure the leaky Rayleigh-type SAW devices. In their studies, they conducted a massive number of experiments and reported the effects of IDT finger width to pitch ratio and electrode thickness on the COM parameters.

In this paper, we present a complete procedure to recover the COM parameters from the measured admittance of a one-port SAW resonator. For completeness, the COM equations, the P-matrix representation and the related COM parameters have been summarized first. The admittance of the one-port resonator was derived based on the Pmatrix representation. One-port and two-port SAW resonator filters were fabricated and the related responses were measured. Then, the simplex method was used to determine the COM parameters inversely. The recovered COM parameters of the one-port SAW resonator have also been used to predict the frequency response of the two-port SAW resonator fabricated under a similar manufacturing process.

\section{Coupling of Modes Model for a Uniform SAW Transducer}

Coupling of modes equations have been derived and utilized for analyzing SAW transducers with constant or arbitrary reflectivity weighting. ${ }^{5,6)}$ In the formulations, the effects of propagation loss, electrode reflections, electrical transduction, acoustic reception, thin film loss and the distributed finger capacitance have been included. In this paper, we adopted the COM formulation presented by Abbott. ${ }^{4)}$ For a uniform transducer, the spatial dependence of the COM parameters disappeared, and the COM equations governing SAW mode amplitudes $R(x, \omega)$, $S(x, \omega)$ propagating in the $\pm x$ directions (Fig. 1) can be arranged in a concise form as ${ }^{4)}$

$$
\begin{aligned}
\frac{d R(x)}{d x}= & -j k_{\mathrm{E}} R(x)+j K_{\mathrm{R}} e^{-j 2 k_{0} x} S(x)+j \alpha_{\mathrm{R}} V_{0} e^{-j k_{0} x} \\
\frac{d S(x)}{d x}= & +j k_{\mathrm{E}} S(x)-j K_{\mathrm{S}} e^{+j 2 k_{0} x} R(x)-j \alpha_{\mathrm{S}} V_{0} e^{+j k_{0} x} \\
\frac{d I(x)}{d x}= & +j 2 \alpha_{\mathrm{S}} R(x) e^{+j k_{0} x}+j 2 \alpha_{\mathrm{R}} S(x) e^{-j k_{0} x} \\
& -j\left(\frac{3 \omega C_{\mathrm{F}} / \Lambda_{\mathrm{T}}}{3+j \omega R_{\mathrm{F}} C_{\mathrm{F}}}\right) V_{0},
\end{aligned}
$$

where $\Lambda_{\mathrm{T}}$ is the wavelength of transduction, $V_{0}$ is the voltage across the IDT, $k_{0}=\frac{2 \pi}{\Lambda_{\mathrm{T}}}$ is the transducer's synchronous wave-number and

$$
k_{\mathrm{E}}=+\frac{\omega}{v_{\mathrm{R}}}-\left(\frac{2 \alpha^{2} \omega C_{\mathrm{F}} R_{\mathrm{F}}^{2} \Lambda_{\mathrm{T}}}{9+\left(\omega R_{\mathrm{F}} C_{\mathrm{F}}\right)^{2}}\right)
$$

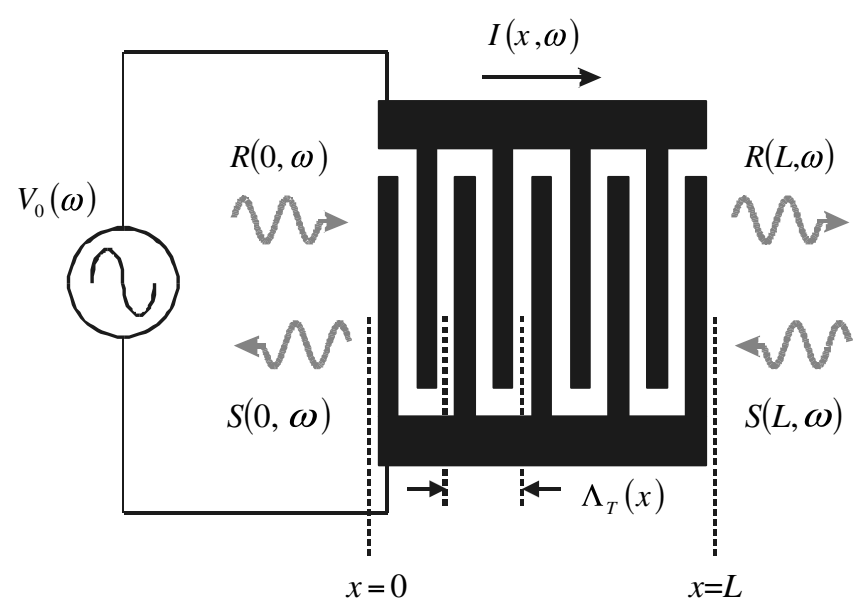

Fig. 1. Coordinates of the IDT. 


$$
\begin{aligned}
& -j\left[\gamma+\left(\frac{6 \alpha^{2} R_{\mathrm{F}} \Lambda_{\mathrm{T}}}{9+\left(\omega R_{\mathrm{F}} C_{\mathrm{F}}\right)^{2}}\right)\right] \\
\alpha_{\mathrm{R}}= & \frac{3 \alpha e^{+j \phi_{\mathrm{T}}}}{3+j \omega R_{\mathrm{F}} C_{\mathrm{F}}} \\
\alpha_{\mathrm{S}}= & \frac{3 \alpha e^{-j \phi_{\mathrm{T}}}}{3+j \omega R_{\mathrm{F}} C_{\mathrm{F}}} \\
K_{\mathrm{R}}= & +K e^{+j \phi_{\mathrm{B}}}+\frac{2 j \alpha^{2} R_{\mathrm{F}} \Lambda_{\mathrm{T}} e^{-j 2 \phi_{\mathrm{T}}}}{3+j \omega C_{\mathrm{F}} R_{\mathrm{F}}} \\
K_{\mathrm{S}}= & +K e^{-j \phi_{\mathrm{B}}}+\frac{2 j \alpha^{2} R_{\mathrm{F}} \Lambda_{\mathrm{T}} e^{+j 2 \phi_{\mathrm{T}}}}{3+j \omega C_{\mathrm{F}} R_{\mathrm{F}}} .
\end{aligned}
$$

In the equations, $\phi_{\mathrm{B}}=\pi / 2$ and $\phi_{\mathrm{T}}=\pi$ are the phase offsets of the grating and the potential, respectively. The origin of the coordinate system is located at a distance $\Lambda_{\mathrm{T}} / 4$ away from the center of the first IDT finger (Fig. 1). The COM parameters are

$\nu_{\mathrm{R}}$ : Rayleigh wave velocity of the substrate,

$\alpha$ : transduction coefficient,

$R_{\mathrm{F}}$ : thin film resistance in one transduction period,

$C_{\mathrm{F}}$ : interdigital capacitance in one transduction period,

$K$ : reflection parameter,

$\gamma$ : propagation loss per unit length.

To facilitate the cascading of uniform transducer elements, solutions of the COM equations can be presented in the $P$ matrix form. In the $P$ matrix representation, the acoustic ports are treated as scattering ports and the electric port as the admittance port as

$$
\left[\begin{array}{c}
S(0) \\
R(L) \\
I
\end{array}\right]=\left[\begin{array}{lll}
P_{11} & P_{12} & P_{13} \\
P_{21} & P_{22} & P_{23} \\
P_{31} & P_{32} & P_{33}
\end{array}\right]\left[\begin{array}{c}
R_{I}(0) \\
S_{I}(L) \\
V_{0}
\end{array}\right],
$$

where

$$
\begin{aligned}
P_{11}= & \frac{+j K_{\mathrm{S}} \sin (D L)}{D \cos (D L)+j \Delta \sin (D L)} \\
P_{12}= & \frac{D}{D \cos (D L)+j \Delta \sin (D L)} e^{-j k_{0} L} \\
P_{13}= & +j L\left(\frac{\sin (D L / 2)}{D L / 2}\right) \\
& \times\left[\frac{\alpha_{\mathrm{S}} D \cos (D L / 2)+j\left(K_{\mathrm{S}} \alpha_{\mathrm{R}}+\Delta \alpha_{\mathrm{S}}\right) \sin (D L / 2)}{D \cos (D L)+j \Delta \sin (D L)}\right] \\
P_{22}= & \frac{+j K_{\mathrm{R}} \sin (D L)}{D \cos (D L)+j \Delta \sin (D L)} e^{-j 2 k_{0} L} \\
P_{23}= & +j L\left(\frac{\sin (D L / 2)}{D L / 2}\right) \\
& \times\left[\frac{\alpha_{\mathrm{R}} D \cos (D L / 2)+j\left(K_{\mathrm{R}} \alpha_{\mathrm{S}}+\Delta \alpha_{\mathrm{R}}\right) \sin (D L / 2)}{D \cos (D L)+j \Delta \sin (D L)}\right] e^{-j k_{0} L}
\end{aligned}
$$

$$
\begin{aligned}
P_{33}= & -j 2\left(\frac{K_{\mathrm{S}} \alpha_{\mathrm{R}}^{2}+K_{\mathrm{R}} \alpha_{\mathrm{S}}^{2}+2 \Delta \alpha_{\mathrm{S}} \alpha_{\mathrm{R}}}{D^{3}}\right) \\
& \times\left[D L-\frac{D \sin (D L)+j \Delta(1-\cos (D L))}{D \cos (D L)+j \Delta \sin (D L)}\right] \\
& -2\left(\frac{\Delta\left(K_{\mathrm{S}} \alpha_{\mathrm{R}}^{2}+K_{\mathrm{R}} \alpha_{\mathrm{S}}^{2}\right)+2 K_{\mathrm{R}} K_{\mathrm{S}} \alpha_{\mathrm{R}} \alpha_{\mathrm{S}}}{D^{3}}\right)
\end{aligned}
$$

$$
\times\left(\frac{1-\cos (D L)}{D \cos (D L)+j \Delta \sin (D L)}\right)+j\left(\frac{3 \omega C_{\mathrm{F}} L / \Lambda_{\mathrm{T}}}{3+j \omega R_{\mathrm{F}} C_{\mathrm{F}}}\right)
$$

and $\Delta=k_{\mathrm{E}}-k_{0}, D=\sqrt{\Delta^{2}-K_{\mathrm{R}} K_{\mathrm{S}}}$.

\section{One-Port SAW Resonator}

In this section, we express the admittance of a one-port SAW resonator in terms of the components of a $P$ matrix. Figure 2 shows schematics of the one-port SAW resonator, which consists of an interdigital transducer for both voltage input and output, and two reflected gratings on both sides. $D_{2}$ and $D_{4}$ are the distances between the IDT and the left grating and right grating, respectively. As an external voltage input to the resonator, the IDT converts electrical signals into surface acoustic waves, which then propagate away from the IDT along the $\pm x$ directions. As the waves hit the metal gratings, they are reflected back to the IDT and form a resonant cavity. To enhance the reflection, the metal gratings are usually in short circuit. In that case, the reflection coefficient $\Gamma$ of the metal gratings can be obtained from the IDT's P matrix equation [eq. (9)] by setting $V_{0}=0$, $R_{\mathrm{F}}(x)=0$ and $C_{\mathrm{F}}(x)=0$. It is easily seen that for wave incidents from the left side of a grating, the reflection coefficient is $P_{11}$, and for wave incidents from the right side of a grating, the reflection coefficient is $P_{22}$.

Utilizing the grating reflection coefficients, section A of the one-port resonator shown in Fig. 3 can be simplified into the form, which consists of a $2 \times 2 P$-matrix as ${ }^{9)}$

$$
\left[\begin{array}{c}
b_{1}^{\mathrm{A}} \\
i_{2}^{\mathrm{A}}
\end{array}\right]=\left[\begin{array}{cc}
P_{11}^{\mathrm{A}} & P_{12}^{\mathrm{A}} \\
P_{21}^{\mathrm{A}} & P_{22}^{\mathrm{A}}
\end{array}\right]\left[\begin{array}{c}
a_{1}^{\mathrm{A}} \\
u_{2}^{\mathrm{A}}
\end{array}\right],
$$

where $\Gamma^{\mathrm{A}}=P_{22} e^{-2 j k D_{2}}$ and

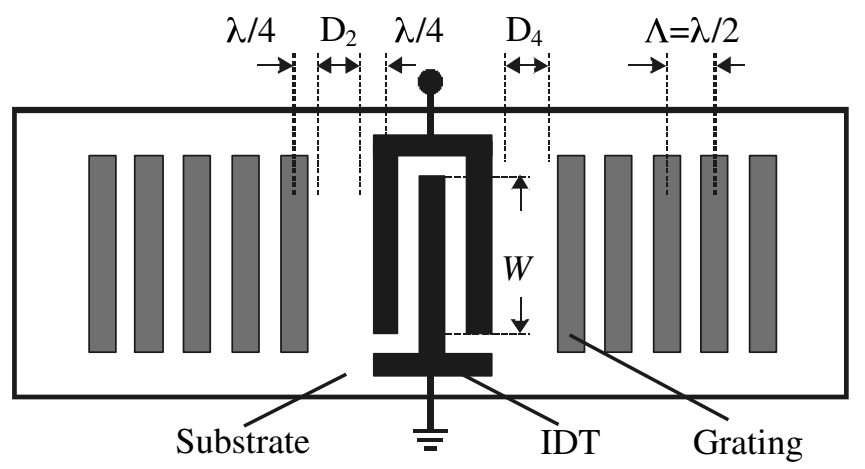

Fig. 2. Schematics of the one-port SAW resonator.

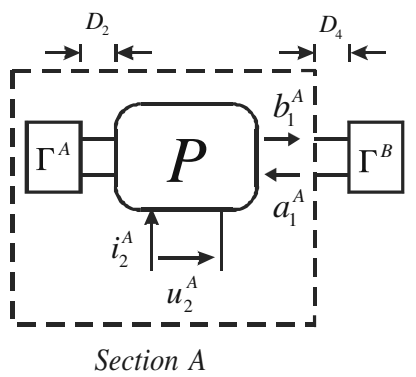

Fig. 3. Simplified block diagram of the one-port SAW resonator. 


$$
\begin{aligned}
& P_{11}^{\mathrm{A}}=P_{22}+\frac{\Gamma^{\mathrm{A}} P_{12} P_{21}}{1-\Gamma^{\mathrm{A}} P_{11}} \\
& P_{12}^{\mathrm{A}}=P_{23}+\frac{\Gamma^{\mathrm{A}} P_{13} P_{21}}{1-\Gamma^{\mathrm{A}} P_{11}} \\
& P_{21}^{\mathrm{A}}=P_{32}+\frac{\Gamma^{\mathrm{A}} P_{12} P_{31}}{1-\Gamma^{\mathrm{A}} P_{11}} \\
& P_{22}^{\mathrm{A}}=P_{33}+\frac{\Gamma^{\mathrm{A}} P_{13} P_{31}}{1-\Gamma^{\mathrm{A}} P_{11}} .
\end{aligned}
$$

In addition, using the reflection from the grating on the right side of section $\mathrm{A}$, we have $a_{1}^{\mathrm{A}}=\Gamma^{\mathrm{B}} b_{1}^{\mathrm{A}}$, where $\Gamma^{\mathrm{B}}=$ $P_{11} e^{-2 j k D_{4}}$. If we substitute $a_{1}^{\mathrm{A}}=\Gamma^{\mathrm{B}} b_{1}^{\mathrm{A}}$ into eq. (16), the relationship between the current $i_{2}^{\mathrm{A}}$ and the voltage $u_{2}^{\mathrm{A}}$ can be written as

$$
i_{2}^{\mathrm{A}}=Y u_{2}^{\mathrm{A}}
$$

where $Y$ is called the admittance of the one-port SAW resonator and is equal to

$$
Y=P_{22}^{\mathrm{A}}+\frac{\Gamma^{\mathrm{B}} P_{12}^{\mathrm{A}} P_{21}^{\mathrm{A}}}{1-\Gamma^{\mathrm{B}} P_{11}^{\mathrm{A}}} .
$$

The real part and the imaginary part of $Y$ are the conductance and susceptance of the resonator, respectively.

\section{Theoretical Calculation of the COM Parameters}

The frequency response of a SAW resonator can be calculated by using the COM-based $\mathrm{P}$ matrix provided that the COM parameters are available. The COM parameters are: surface wave velocity $v_{\mathrm{R}}$, reflection parameter $K$, propagation loss $\gamma$, transduction parameter $\alpha$, thin film finger capacitance $C_{\mathrm{F}}$ and finger resistance $R_{\mathrm{F}}$. In the following, the formulae or values we used in this study are given.

\subsection{Surface wave velocity $v_{R}$}

The perturbation of surface wave velocity by a thin metal film grating was calculated using the free software FEMSDA developed by Hashimoto and Yamaguchi. ${ }^{10)}$

\subsection{Transduction parameter $\alpha$}

The transduction coefficient $\alpha$, which is responsible for the excitation efficiency of the IDT, can be derived as ${ }^{11)}$

$$
\alpha(\omega)=\frac{Q_{\mathrm{F}}(k)}{\Lambda_{\mathrm{T}}} \sqrt{\frac{\omega W \Gamma_{\mathrm{S}}}{2}}
$$

with the elemental charge density $Q_{\mathrm{F}}$ defined as

$$
\begin{gathered}
Q_{\mathrm{F}}(k)=\varepsilon_{\mathrm{S}}(\infty) \frac{2 \sin (\pi s)}{P_{-s}(-\cos \eta)} P_{m}(\cos \eta) \\
\text { for } m \leq \frac{k p}{2 \pi} \leq m+1,
\end{gathered}
$$

where $W$ is the aperture of the IDT, $\eta=\pi a / p$ and $a / p$ is the electrode width to grating period ratio, $\varepsilon_{S}(\infty)$ is the surface effective permittivity as a function of the slowness, $m$ is an integer, $s=\frac{k p}{(2 \pi)}-m, P_{-s}(-\cos \eta)$ is a Legendre function and $P_{m}(\cos \eta)$ is a Legendre polynomial. The coefficient $\Gamma_{\mathrm{S}}$ is defined as ${ }^{11)}$

$$
\frac{1}{\Gamma_{s}}=-k_{0}\left[\frac{d \varepsilon_{\mathrm{S}}(k)}{d k}\right]_{k_{0}} .
$$

\subsection{Reflection parameter $K$}

The reflection parameter $K$ represents the reflectivity of the thin film finger in the IDT or grating. It arises from two causes: the electrical loading and the mechanical loading, and can be expressed by ${ }^{12,13)}$

$$
K=\left[R_{\mathrm{k}}\left(\frac{k_{\mathrm{e}}^{2}}{2}\right)+R_{\mathrm{m}}\left(\frac{h}{\lambda}\right) \sin \left(\frac{a \pi}{p}\right)\right] \frac{1}{p},
$$

where $R_{\mathrm{k}}$ and $R_{\mathrm{m}}$ denote the electrical effect and mechanical effect, respectively. $h$ is the metal film thickness, $\lambda$ is the wavelength of the surface wave and $k_{\mathrm{e}}^{2}$ is the electromechanical coupling coefficient. The functions $R_{\mathrm{k}}$ and $R_{\mathrm{m}}$ are

$$
\begin{aligned}
R_{\mathrm{k}}= & -\frac{\pi}{2}\left[\cos (\pi \eta)+\frac{P_{s}[-\cos (\pi \eta)]}{P_{s-1}[-\cos (\pi \eta)]}\right] \\
R_{\mathrm{m}}= & -\frac{\pi k_{\mathrm{e}}^{2}}{\varepsilon_{\mathrm{S}}(\infty)}\left[\left(\frac{U_{1}}{\varphi}\right)^{2}\left(\alpha_{1}+\rho^{\prime} v_{\mathrm{f}}^{2}\right)\right. \\
& \left.+\left(\frac{U_{2}}{\varphi}\right)^{2}\left(\alpha_{2}+\rho^{\prime} v_{\mathrm{f}}^{2}\right)+\left(\frac{U_{3}}{\varphi}\right)^{2} \rho^{\prime} v_{\mathrm{f}}^{2}\right],
\end{aligned}
$$

where $U_{1} U_{2} U_{3}$ are the displacements of the surface wave, $\varphi$ the surface electrical potential, $\rho^{\prime}, \lambda^{\prime}, \mu^{\prime}$ are the density and Lame constants of the thin film electrode, and

$$
\alpha_{1}=\frac{4 \mu^{\prime}\left(\lambda+\mu^{\prime}\right)}{\lambda^{\prime}+2 \mu^{\prime}}, \quad \alpha_{2}=\mu^{\prime} .
$$

The electromechanical coefficient $k_{\mathrm{e}}^{2}$ can be obtained from ${ }^{14)}$

$$
k_{\mathrm{e}}^{2}=2 \Gamma_{s} \varepsilon_{s}^{(\infty)} .
$$

\subsection{Thin film finger resistance $R_{F}$}

For a single electrode type IDT, the thin film finger resistance can be determined by ${ }^{11)}$

$$
R_{\mathrm{F}}=2 \rho W / 3 a N_{\mathrm{p}},
$$

where $\rho$ is the sheet resistance of a metal film, $W$ is the aperture, $a$ is the finger width and $N_{\mathrm{p}}$ the number of IDT pairs. For an aluminum thin film with thickness $h(\mu \mathrm{m})$ between $0.05 \mu \mathrm{m}$ to $0.3 \mu \mathrm{m}$, the sheet resistance can be approximated as

$$
\rho \approx 0.04 / h \quad(\Omega / \text { square }) .
$$

\subsection{Thin film finger capacitance $C_{F}$}

For ST-X quartz with an aluminum finger on top and the finger width to pitch ratio $a / p=0.5$, the finger capacitance can be approximated as. ${ }^{15)}$

$$
C_{0}=0.503 \quad(\mathrm{pF} / \mathrm{cm}) .
$$

\subsection{Propagation loss $\gamma$}

For ST-X quartz, the propagation loss can be approximated as ${ }^{11)}$

$$
\gamma \approx 0.47 f+2.62 f^{2} \quad(\mathrm{~dB} / \mu \mathrm{s})
$$

where the operating frequency $f$ is in $\mathrm{GHz}$.

On employing eq. (22) and the COM parameters described in this section, the admittance of a one-port resonator can be calculated. Shown in Fig. 4 is the result of simulated conductance and susceptance of a one-port 


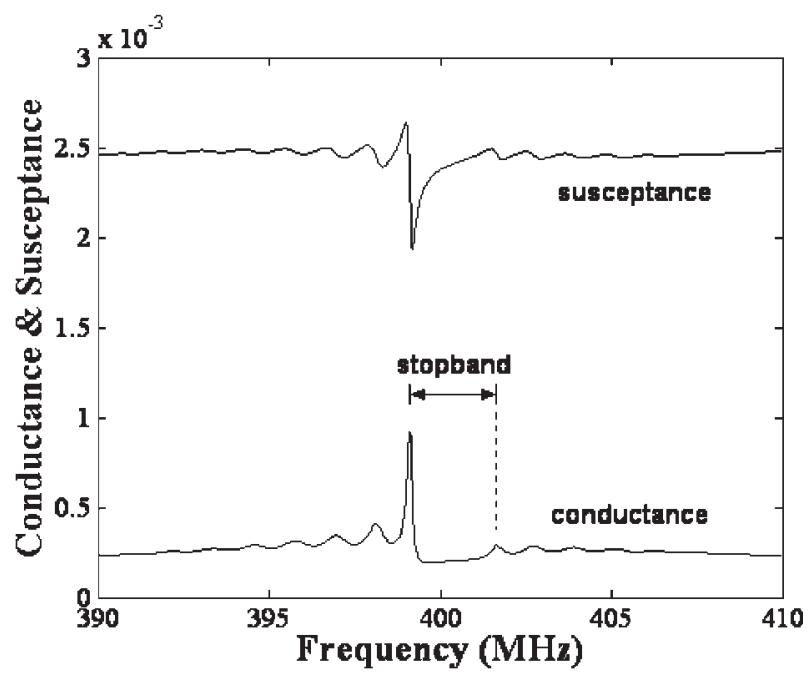

Fig. 4. Simulated conductance and susceptance of a one-port synchronous SAW resonator.

synchronous SAW resonator. The substrate of the resonator is ST-quartz, the period of the fingers is $p=3.932 \mu \mathrm{m}$, the aperture is $W=100 * 2 p$, the number of IDT pairs is $N_{\mathrm{t}}=25$, and the number of gratings is $N_{\mathrm{g}}=300$. Parametric simulation results revealed that the position of the stopband is predominantly controlled by the surface wave velocity $\nu_{R}$, and the width of the stopband is controlled by the reflection parameter $K$. The amplitudes of the conductance and susceptance are influenced by the transduction parameter $\alpha$ and the propagation loss $\gamma$. The thin film resistance $R_{\mathrm{F}}$ and capacitance $C_{\mathrm{F}}$ are related to the DC shift of the conductance and susceptance curves.

\section{Experimental}

A one-port synchronous SAW resonator similar to the schematics shown in Fig. 2 was fabricated. The substrate of the resonator was ST-quartz, the period of the fingers was $p=3.932 \mu \mathrm{m}$, the aperture was $W=100 * 2 p$, the number of IDT pairs was $N_{\mathrm{t}}=50$, and the number of gratings was $N_{\mathrm{g}}=300$. The aluminum thin film thickness was $h=$ $160 \mathrm{~nm}$, and the finger width to pitch ratio was $m / p=0.5$. The solid lines shown in Figs. 5 and 6 are the measured conductance and susceptance of the resonator, respectively. The measurements were conducted through the utilization of a wafer probe. For later comparison, a standard two-port resonator filter was also fabricated in the same wafer. The material of the substrate and the number of IDT pairs and gratings are all the same as those of the one-port SAW resonator. The distance between the grating and the IDT was $3 / 8$ wavelength and the delay of the two IDTs was 15 wavelength. The solid line shown in Fig. 7 denotes the measured insertion loss of the two-port resonator filter. On comparison with the calculated insertion loss (dotted line), a downshift of the center frequency was found.

\section{Inversion of the COM Parameters}

In previous sections, we have shown that the conductance and susceptance of a one-port SAW resonator can be calculated using the P-Matrix formulation. We have also demonstrated that the six COM parameters affect the characteristics of the one-port resonator's admittance in

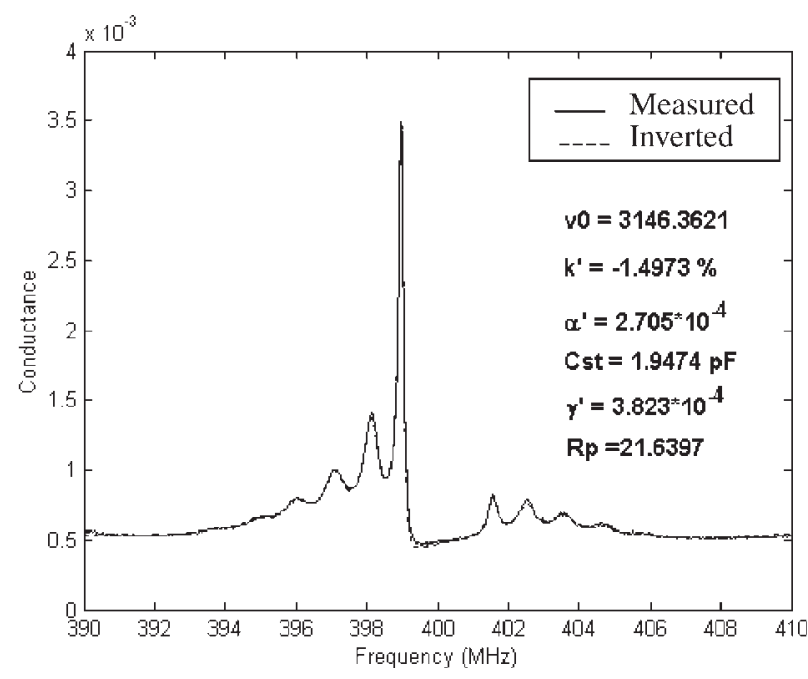

Fig. 5. Measured conductance of the one-port synchronous SAW resonator.

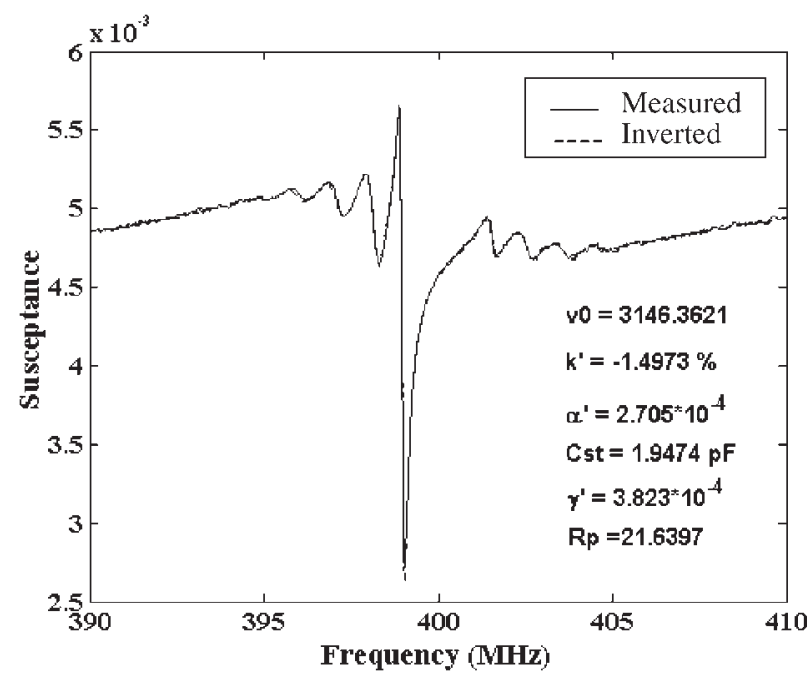

Fig. 6. Measured susceptance of the one-port synchronous SAW resonator.

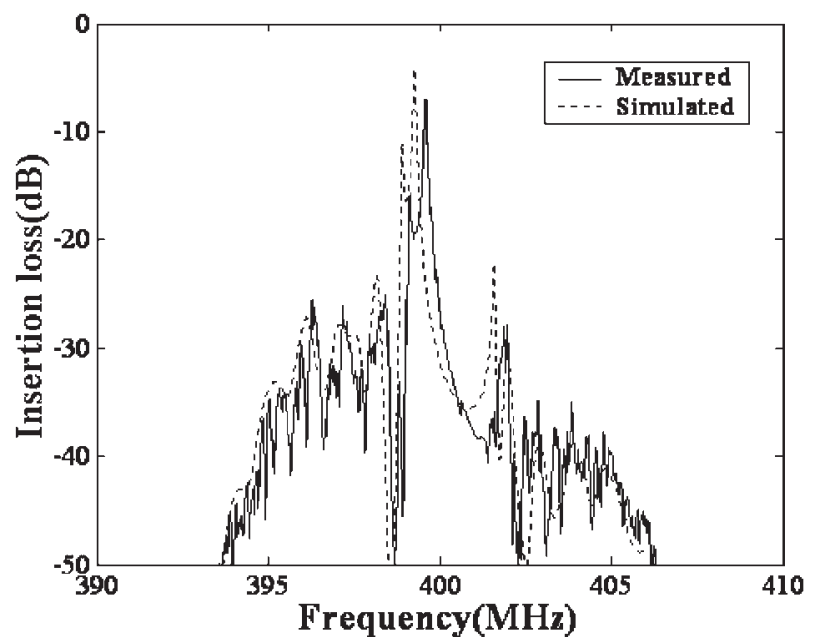

Fig. 7. Measured (solid line) and simulated (dotted line) insertion loss of the two-port resonator filter. 
various ways. For example, the surface wave velocity $v_{R}$ affects the center frequency of the stopband and the reflection parameter $K$ affects the bandwidth of the stopband. On having the forward simulation model and the measured admittance, the COM parameters can be determined inversely through the utilization of an inverse algorithm.

In this study, we adopted the downhill simplex method $^{16,17)}$ as the inverse optimization algorithm. We note that this method is totally different from the simplex method in linear programming, the down hill method can be used in the nonlinear optimization problems. ${ }^{18)}$ An error function $e$, which is proportional to the absolute value of the complex admittance, was defined as

$$
e=\sum_{i=1}^{N}\left\{\left[C_{\mathrm{m}}(i)-C_{\mathrm{g}}(i)\right]^{2}+\left[S_{\mathrm{m}}(i)-S_{\mathrm{g}}(i)\right]^{2}\right\}
$$

where $C_{\mathrm{m}}, S_{\mathrm{m}}$ are the measured conductance and susceptance and $C_{\mathrm{g}}, S_{\mathrm{g}}$ are the guessed conductance and susceptance. $i$ represents the discrete frequency and $N$ is the number of data points utilized in the inversion process. In the inversion process using the simplex method, if there are $n$ unknowns to be recovered, then, $n+1$ sets of unknown vectors have to be guessed initially. To speed up the inversion efficiency and avoid the local minimum, initial guesses of the COM parameters are important. Furthermore, imposed constraints on the COM parameters are also important for obtaining a correct result.

In the following, we explain the guidelines for making the initial guesses and the necessary constraints. We used the center frequency of the stopband in the measured admittance curve to estimate the surface wave velocity and serve as the initial guess. The upper and lower margin frequencies of the stopband were used to estimate the constraints of the surface wave velocity. As for the reflection parameter, we use the information carried by the bandwidth of the stopband that the larger the reflection parameter, the bigger the width of the stopband.

Parametric simulation results on the effect of finger resistance and capacitance on the admittance of a one-port resonator have shown that they only caused the DC shift of the admittance curves. The position and bandwidth of the stopband are not influenced by their changes. The resistance of a one-port resonator consists of the finger resistance, the resistance of the bus bar electrode and the contact resistance. These effects cannot be decoupled easily from a single admittance measurement. In this paper, instead of recovering the finger resistance $R_{\mathrm{F}}$, we recovered the total resistance $R_{\mathrm{P}}$ of the resonator. This was done by setting $R_{\mathrm{F}}=0$ in the COM model first, and then, added a total resistance $R_{\mathrm{P}}$ in series with the impedance of the resonator ${ }^{19)}$ as shown in Fig. 8.

As for the initial guess of the transduction parameter $\alpha$, the propagation loss $\gamma$ and the thin film capacitance $C_{\mathrm{F}}$, results showed that the values calculated by the aforementioned COM model could serve as good initial guess. Numerical experience has shown that as long as those initial guesses are in the same order of the theoretical calculated one, a stable inversion can be achieved.

In the inversion process, the COM parameters have been

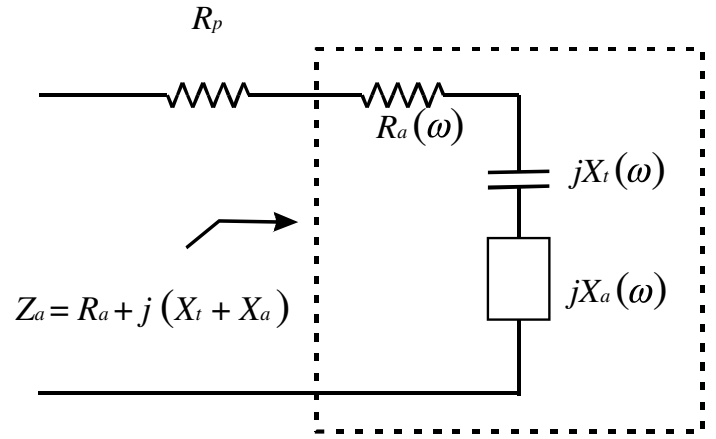

Fig. 8. Simplified equivalent circuit of the one-port resonator.

Table I. Guessed and recovered COM parameters from the one-port SAW resonator admittance.

\begin{tabular}{|c|c|c|c|c|c|}
\hline \multicolumn{6}{|c|}{ Initial guess } \\
\hline$v_{0}(\mathrm{~m} / \mathrm{s})$ & $\kappa^{\prime}(\%)$ & $\alpha^{\prime}(1 / \sqrt{\Omega} \lambda)$ & Cst $(\mathrm{pF})$ & $\gamma^{\prime}($ neper $/ \lambda)$ & $R_{\mathrm{P}}(\Omega)$ \\
\hline 3146.1 & -1.5 & $2.98 \times 10^{-4}$ & 1.8878 & $2.96 \times 10^{-4}$ & 16 \\
\hline 3147.2 & -1.95 & $2.58 \times 10^{-4}$ & 1.4565 & $3.34 \times 10^{-4}$ & 15 \\
\hline 3146.4 & -1.05 & $2.62 \times 10^{-4}$ & 2.1785 & $4.24 \times 10^{-4}$ & 17 \\
\hline 3146.3 & -1.385 & $3.54 \times 10^{-4}$ & 2.2388 & $3.07 \times 10^{-4}$ & 20 \\
\hline 3146.5 & -1.67 & $1.93 \times 10^{-4}$ & 2.038 & $5.57 \times 10^{-4}$ & 25.5 \\
\hline 3146 & -1.165 & $2.68 \times 10^{-4}$ & 1.3475 & $2.10 \times 10^{-4}$ & 21 \\
\hline 3145.9 & -1.47 & $2.29 \times 10^{-4}$ & 2.5691 & $4.05 \times 10^{-4}$ & 12.5 \\
\hline \multicolumn{6}{|c|}{ Inversed result } \\
\hline$v_{0}(\mathrm{~m} / \mathrm{s})$ & $\kappa^{\prime}(\%)$ & $\alpha^{\prime}(1 / \sqrt{\Omega} \lambda)$ & Cst $(\mathrm{pF})$ & $\gamma^{\prime}($ neper $/ \lambda)$ & $R_{\mathrm{P}}(\Omega)$ \\
\hline 3146.3621 & -1.4973 & $2.705 \times 10^{-4}$ & 1.9474 & $3.823 \times 10^{-4}$ & 21.6397 \\
\hline
\end{tabular}

redefined as $\kappa^{\prime}=K * \lambda$, the reflectivity of electrode system per wavelength, $\gamma^{\prime}=\gamma * \lambda$, the attenuation per wavelength, $\alpha^{\prime}=\alpha * \lambda$, the electromechanical coupling constant, and $C s t=C_{\mathrm{F}} * N_{\mathrm{t}}$, the static capacitance of the transducer. The initial guesses and the recovered COM parameters are listed in Table I. The dotted lines in Figs. 5 and 6 are the conductance and susceptance curves calculated based on the inversely determined COM parameters. We note that the measured and the inversely calculated results were in perfect agreement. Except for the surface wave velocity, we note that the initial guesses of the COM parameters can be about $30 \%$ different from the true values.

In the following, we show that the COM parameters determined inversely from the one-port resonator can be utilized in the design of a two-port SAW resonator as well. The dotted line in Fig. 7 denotes the calculated insertion loss of the two-port SAW resonator filter. The COM parameters were calculated based on the formulae (or values) given in $\S 4$. Although the basic characteristics of the two-port SAW resonator filter has been predicted by the COM simulation, a frequency shift exists between the measured and simulated insertion loss. The dotted line in Fig. 9 denotes the simulated insertion loss based on the COM parameters inversely determined from the one-port resonator. In this case, we note that the measured and the simulated resonant peaks of the insertion loss match quite well.

\section{Conclusion}

Extraction of the COM parameters from the SAW manufacturing process is important in practice. In this 


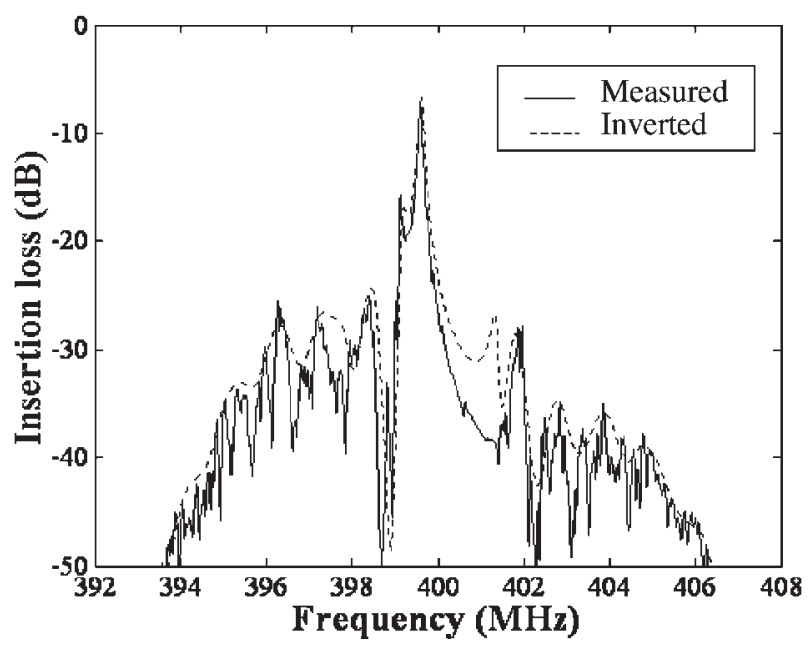

Fig. 9. Simulated insertion loss (dotted line) based on the COM parameters inversely determined from the one-port resonator.

paper, we have presented an inverse method for recovering the COM parameters from the admittance measurement. To serve as the forward simulation of the admittance of the oneport resonator, the COM equations, the P-matrix representation and the related COM parameters have been summarized briefly. One-port and two-port SAW resonator filters were fabricated through the standard lithography process and the related responses were measured. The simplex method has been used to determine the COM parameters inversely and the results are in agreement with the standard values. The recovered COM parameters have also been used to calculate the frequency response of the two-port SAW resonator. Results have shown that the calculated response well matches the measured one.

\section{Acknowledgment}

The authors acknowledge the financial support for this research provided by National Science Council of Taiwan (NSC90-2212-E-002-156) and TXC corporation.

1) P. S. Cross and R. V. Schmidt: Bell Syst. Tech. J. 56 (1977) 1447.

2) P. V. Wright: Ph. D. Thesis, Department of Electrical Engineering, MIT, USA, 1981.

3) D. P. Chen and H. A. Haus: IEEE Trans. Sonics Ultrason. 32 (1985) 395.

4) B. P. Abbott: Ph. D. Thesis, Department of Electrical Engineering, University of Central Florida Orlando, Florida, USA, 1989.

5) P. V. Wright: Proc. 43rd Ann. Frequency Control Symp. (1989) p. 596.

6) B. P. Abbott, C. S. Hartmann and D. C. Malocha: IEEE Ultrasonics Symp. (1989) p. 129.

7) C. S. Hartmann and R. T. Hartmann: IEEE Ultrasonics Symp. (1990) p. 117.

8) C. S. Hartmann and V. P. Plessky: IEEE Ultrasonics Symp. (1993) p. 1247.

9) R. B. Brown, B. H. Horine and J. H. Hines: IEEE Ultrasonics Symp. (1989) p. 153.

10) K. Hashimoto and M. Yamaguchi: Proc. 1996 Frequency Control Symp. (1996) p. 300.

11) D. P. Morgan: Surface-Wave Devices for Signal Processing (Elsevier, New York, 1991).

12) T. Thorvaldsson: IEEE Ultrasonics Symp. (1989) p. 91.

13) T. Thorvaldsson and B. P. Abbott: IEEE Ultrasonics Symp. (1990) p. 43.

14) H. Matthews: Surface Wave Filters: Design, Construction, and Use (Wiley, New York, 1977).

15) C. K. Campbell: Surface Acoustic Wave Devices for Mobile and Wireless Communications (Academic Press, New York, 1998).

16) J. A. Nelder and R. Mead: Comput. J. 7 (1965) 308.

17) T.-T. Wu and Y.-H. Liu: Ultrasonics 37 (1999) 23.

18) W. H. Press, S. A. Teukolsky, W. T. Vetterling and B. P. Flannery: Numerical Recipes (Cambridge University Press, New York, 1992).

19) J. H. Hines and D. C. Malocha: IEEE Ultrasonics Symp. (1993) p. 173. 